\title{
Analysis of the Effect of Different Combinations of Observation Satellites on the Resolving Accuracy of GNSS Observation Data
}

\author{
Junze Wang ${ }^{1}$, Maohua $\mathrm{Yao}^{1}$, Wenting Zhou ${ }^{2, *}$, Xiangping Chen ${ }^{1}$ \\ ${ }^{1}$ Natural Resources Information Center of Guangxi Zhuang Autonomous Region, Nanning, Guangxi, 530023, China \\ ${ }^{2}$ Guilin University of Technology at Nanning, Department of Civil and Surveying Engineering, Chongzuo, Guangxi, 530023, China
}

\begin{abstract}
In this paper, $24 \mathrm{C}$-level control points under different terrain conditions were selected to be the testing points. The binary-satellite system (GPS+GLONASS) and the triple-satellite system with BeiDou Navigation Satellite System (BDS) (BDS+GPS+GLONASS) were adopted for static measurement; and the observation data from BeiDou Ground-based Augumentation System (GBAS) base stations in Guangxi were collected for solution. By comparing the residuals of GPS tri-dimensional baseline vectors and the internal accord accuracy of each control point under the binary and triple-satellite systems, the effect of data collected by different satellite systems under different terrain conditions on measurement accuracy was studied. According to the results, (1) the triple-satellite system with BDS showed more stable measurement accuracy; (2) in plane, the two systems were of equivalent measurement accuracy in mountainous and flat areas; in elevation, the triple-satellite system showed higher and more stable measurement accuracy.
\end{abstract}

\section{Introduction}

China has launched the last of its domestically developed Beidou (BDS) 3 global networking satellites, the networking was completed, which constituted four major global navigation satellite systems (GNSS) ${ }^{[5,7]}$ with the U.S. global positioning system (GPS), the Russian Glonass positioning system (GLONASS), and the European Galileo system (GALILEO) ${ }^{[3-4]}$. Successful networking of BDS marked large-scale application of high-precision real-time navigation and positioning technology across the country supported by BeiDou base stations. At the end of 2018, Guangxi completed upgrading of 102 BeiDou base stations of the whole region based on the original continuous operational reference system (CORS) for satellite positioning (hereinafter referred to as the binary system) ${ }^{[6]}$, introducing BeiDou satellite receivers and building GBAS covering the whole area (hereinafter referred to as the triple-satellite system) to provide users with highprecision, round-the-clock and real-time dynamic positioning service ${ }^{[2]}$. To study the positioning accuracy of the upgraded triple-satellite system, in this paper, 24 C-level control points were included and observed under different system modes; observed data then went through baseline computing and adjustment with Trimble Business Center (TBC) and CORSGPS V6.0 to study the effect of the introduction of BeiDou on positioning accuracy under different satellite navigation systems.

\section{Example Analysis}

\subsection{Data Sources and Processing}

In this paper, 24 C-level control points of known coordinates in the area were selected as subjects, the testing area being in $21^{\circ} 35^{\prime}-24^{\circ} 33^{\prime} \mathrm{N}, 106^{\circ} 53^{\prime}-108^{\circ} 57^{\prime}$ E. Among them, 11 control points (A1, A2, ... A11) were in mountainous areas of complex terrain, where positioning signals and receiving conditions were easily affected; 13 points (A12, A13, ... A24) were in flat areas. 24 control points were measured under the BDS+GPS+GLONASS mode and the GPS+GLONASS mode respectively for the observation time of $4 \mathrm{~h}$ at the data sampling interval of $5 \mathrm{~s}$. Observed data from selected Guangxi BeiDou GBAS base stations, including JZ07, JZ10, JZ11, JZ12, JZ13, JZ14, JZ36, JZ37, JZ45, JZ46, JZ49, JZ50, JZ51, JZ57, JZ64, JZ69, JZ70 and JZ76, were adopted for resolving.

Served data from 24 testing points were collected for baseline computing and adjustment with TBC and CORSGPS V6.0. The resolving process included data import, automatic elimination of gross baseline errors, automatic baseline computing, automatic adjustment, etc., as shown in Figure 1. After baseline resolving, the residuals of GPS tri-dimensional baseline vectors were compared under the binary and the triple-satellite systems; formula (1) [1] shows the evaluation precision:

$$
\left\{\begin{array}{l}
|\mathrm{Vx}| \leq 3 \sigma \\
|\mathrm{Vy}| \leq 3 \sigma \\
|\mathrm{Vz}| \leq 3 \sigma
\end{array}\right.
$$

\footnotetext{
* Corresponding author: 840062819@qq.com
} 
In the formula, $\mathbf{V x}$ is the $\mathrm{X}$-direction residual of the tri-dimensional vector, ${ }^{\mathrm{V}} \mathrm{y}$ the $\mathrm{Y}$-direction residual, and $\mathrm{Vz}$ the Z-direction residual; $\sigma$ is the baseline accuracy for this level.

Based on difference between the resolved threedimensional coordinates and the known true values of 24 C-level control points, the internal accord accuracy of the control points was obtained; formula (2) [1] is the computing formula:

$$
\mu= \pm \sqrt{[V V] /(n-1)}
$$

In which, ${ }^{\mu}$ is internal accord accuracy; $\mathrm{n}$ is the number of control points; $[V V]$ is the square of the difference between the resolved value and the true value.

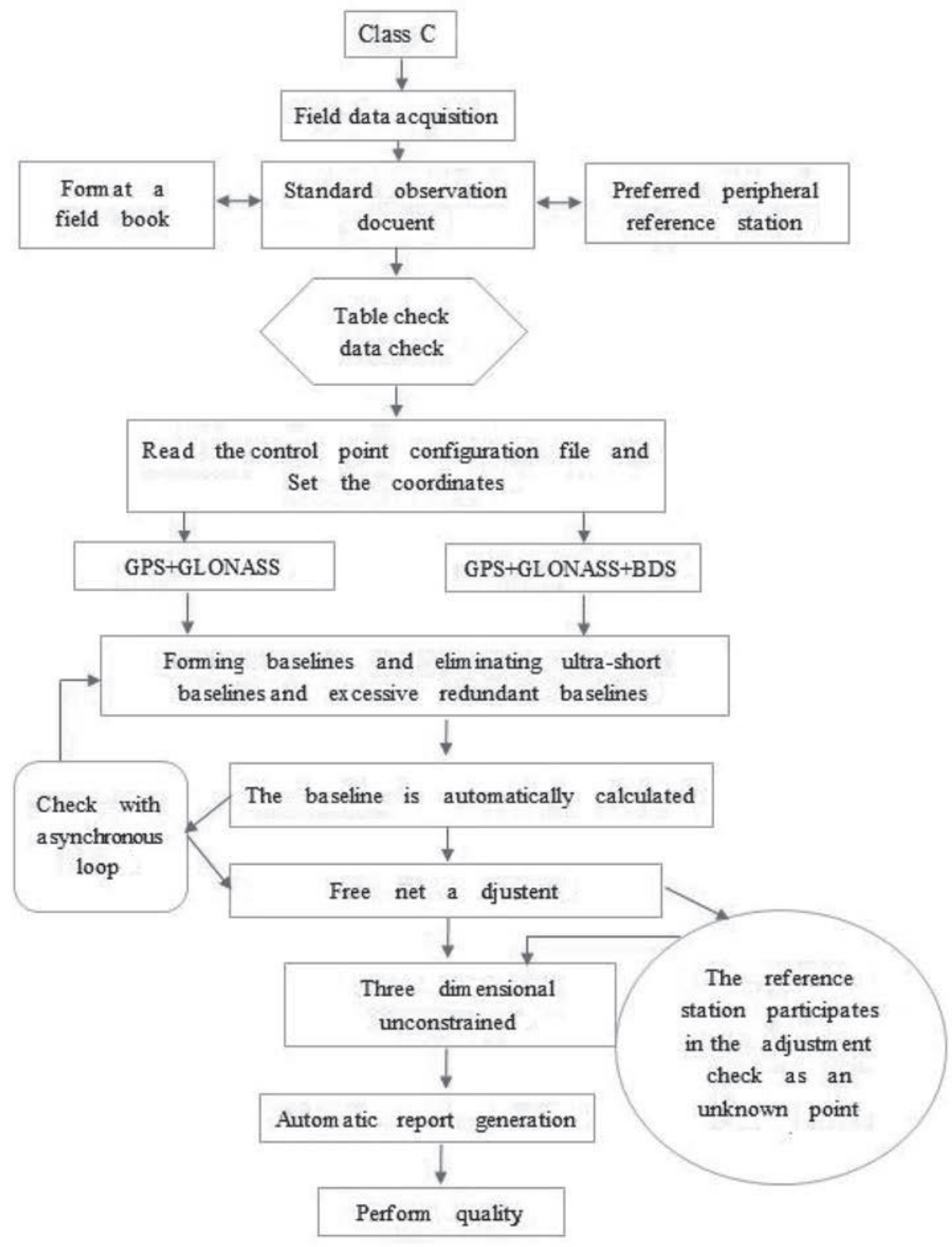

Fig. 1. Data processing flow

\subsection{Accuracy Analysis}

To study the effect of the number of observation satellites on resolving accuracy under different system modes, after the independent-baseline GNSS space vector network consisting of the $24 \mathrm{C}$-level testing points passed the quality check of TBC, data from 24 testing points under different modes were adjusted with CORSGPS V6.0 in CGCS2000 coordinates, thus obtaining the statistical chart of residuals of GPS tridimensional baseline vectors under the binary and triplesatellite systems, as shown in Figure 2:

According to Figure 2 (a), (b) and (c), residuals of GPS tri-dimensional baseline vectors under the triplesatellite system showed higher precision than under the binary system; the precision of residuals was higher in $\mathrm{X}$ and $\mathrm{Y}$ directions than in $\mathrm{Z}$ direction in both systems; the maximum residual of the binary and triple-satellite 
systems in $\mathrm{X}$ and $\mathrm{Y}$ directions was $0.15 \mathrm{~cm}$ approx., and $0.25 \mathrm{~cm}$ approx. in $\mathrm{H}$ direction.

Based on difference between the adjusted threedimensional coordinates and the known true values of 24 control points, the three-dimensional deviation of each control point in different areas was obtained as shown in Figure 3 (a) and Figure 3 (b). The internal accord accuracy of each point was then calculated and collected according to different accuracy ranges, as in Table 1.
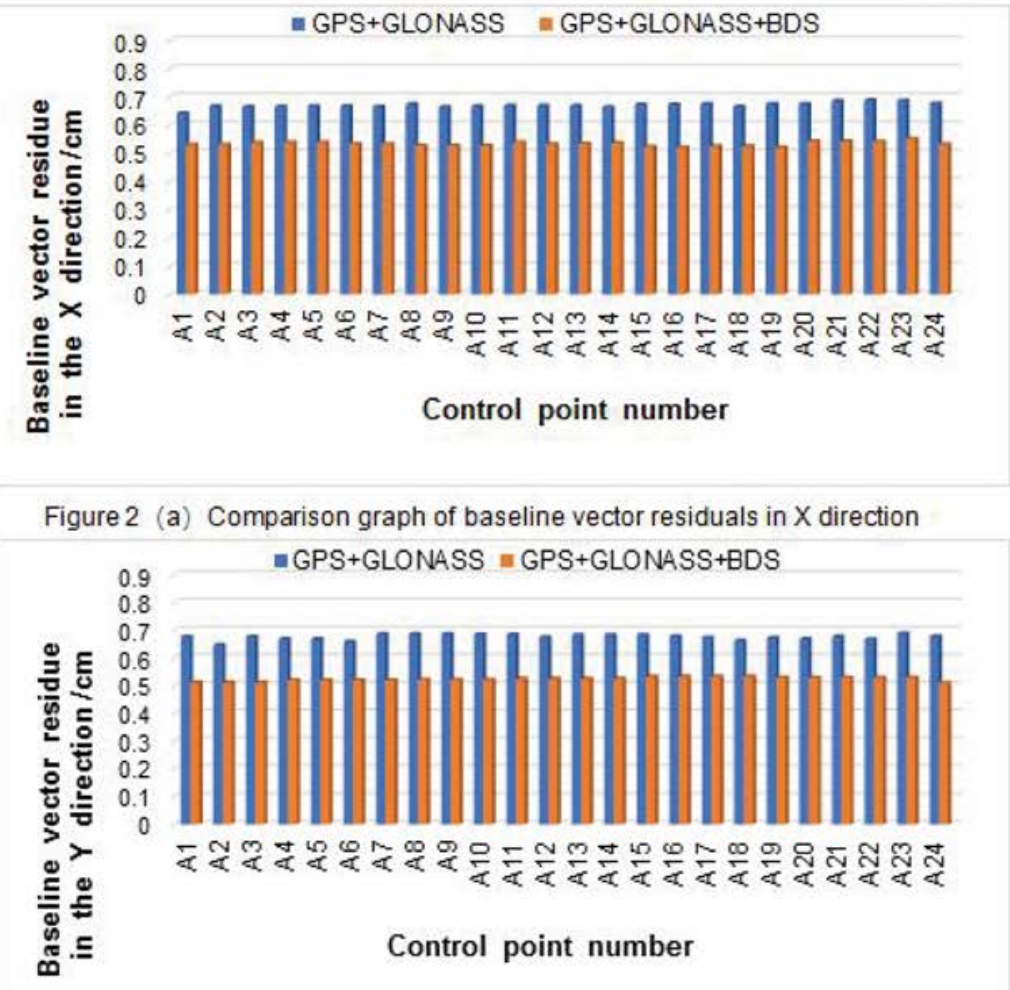

Figure 2 (b) Comparison graph of baseline vector residuals in $\mathrm{Y}$ direction

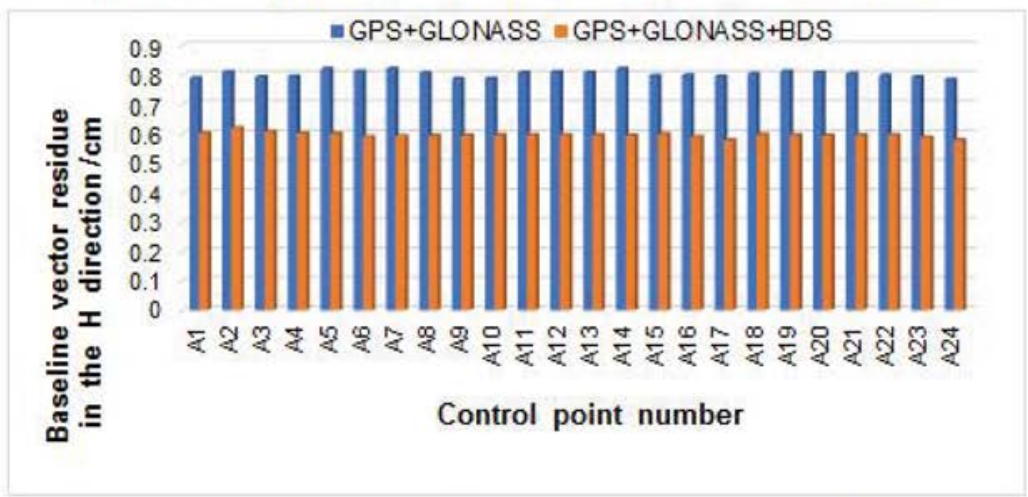

Figure 2 (c) Comparison graph of baseline vector residuals in $\mathrm{H}$ direction

Fig. 2. Comparison of residual values of GPS+GLONASS And GPS+GLONASS+BDS 3D baseline vectors X, Y, and H 


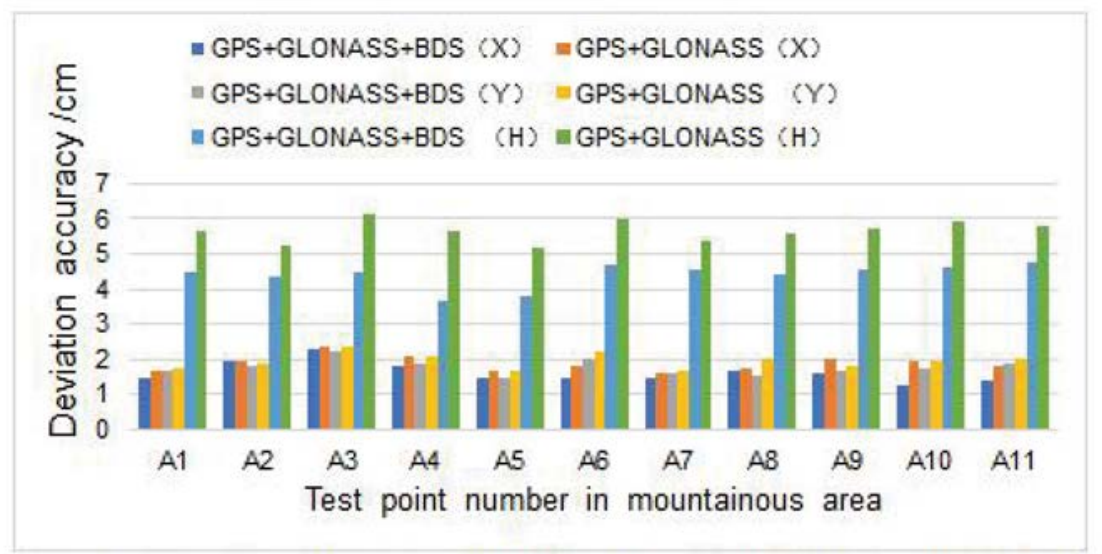

Figure 3 (a) Comparison diagram of test point deviationvalues in mountainous area

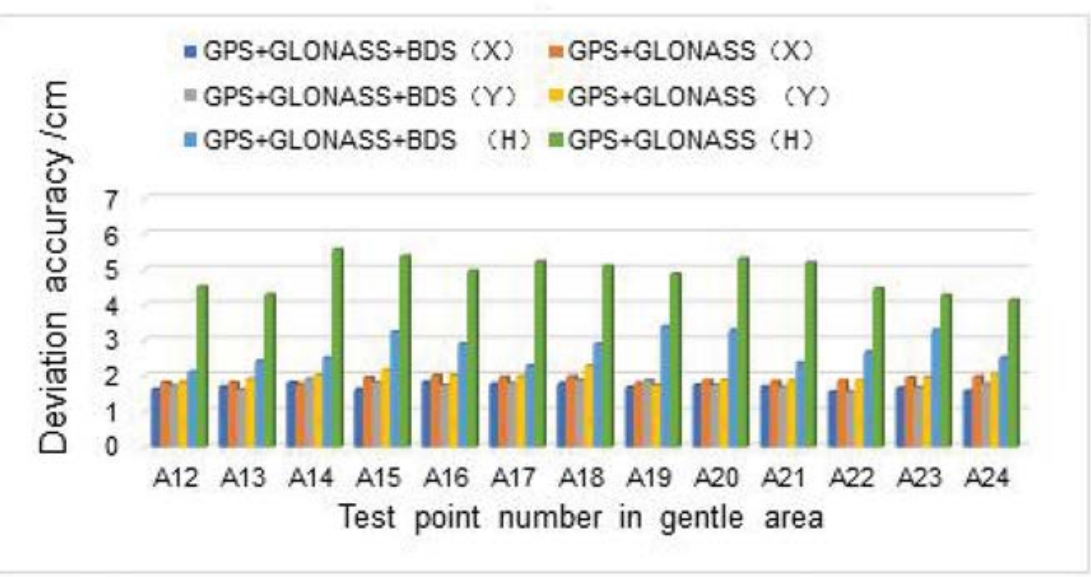

Figure 3 (b) Comparison chart of test point deviation values in gentle region

Fig. 3. Statistical chart of $\mathrm{X}, \mathrm{Y}$ and $\mathrm{H}$ coordinate deviation values Between mountainous region and gentle region

According to Figure 3 (a) and Figure 3 (b), in plane, measured values of the two systems showed little difference from the true values, and received little impact from terrain conditions, i.e. the plane coordinates were close in flat and mountainous areas; in elevation, the triple-satellite system showed significantly smaller deviation than the binary system, i.e. the triple-satellite system better matched the true values, even more obvious in complex mountainous areas. In low relief areas, the maximum elevation was $5.51 \mathrm{~cm}$ under the binary system, and $3.21 \mathrm{~cm}$ under the triple-satellite system; in mountainous areas, the maximum elevation was $6.11 \mathrm{~cm}$ under the binary system, and $4.70 \mathrm{~cm}$ under the triple-satellite system. Elevation deviation of the triple-satellite system in low relief areas was $\pm 2-3.5 \mathrm{~cm}$, the maximum difference between the elevation coordinates of the binary system being $3.2 \mathrm{~cm}$.

Table 1 Absolute precision statistics of $\mathrm{X}, \mathrm{Y}$ and $\mathrm{H}$ of GPS+GLONASS and GPS+GLONASS+BDS

\begin{tabular}{|c|c|c|c|c|c|c|c|}
\hline \multirow{2}{*}{$\begin{array}{c}\text { Measurement } \\
\text { model }\end{array}$} & $\begin{array}{c}\text { Absolute } \\
\text { accuracy range } \\
\text { value /cm }\end{array}$ & \multicolumn{2}{|c|}{$\mathrm{X}$} & \multicolumn{2}{|c|}{ Y } & \multicolumn{2}{c|}{$\mathrm{H}$} \\
\cline { 3 - 8 } & $>5$ & 0 & 0.0 & 0 & 0.0 & 0 & 0.0 \\
\hline \multirow{3}{*}{ GPS } & $3-5$ & 2 & 8.3 & 3 & 12.5 & 2 & 8.3 \\
GLONASS & $2-3$ & 7 & 29.1 & 5 & 20.8 & 14 & 58.3 \\
BDS & $1-2$ & 13 & 54.1 & 9 & 37.5 & 6 & 25.0 \\
& $0-1$ & 2 & 8.3 & 7 & 29.1 & 2 & 8.3 \\
& $>5$ & 0 & 0.0 & 0 & 0.0 & 5 & 20.8 \\
& $3-5$ & 4 & 16.7 & 3 & 12.5 & 10 & 41.7 \\
GPS & $2-3$ & 14 & 58.3 & 10 & 41.7 & 7 & 29.2 \\
GLONASS & $1-2$ & 4 & 16.7 & 6 & 25.0 & 1 & 4.2 \\
& $0-1$ & 2 & 8.3 & 5 & 20.8 & 1 & 4.2 \\
\hline
\end{tabular}


According to the deviation statistics in Table 1, under the triple-satellite system, differences between the measured value and true value of each point concentrated in $2-3 \mathrm{~cm}$ mostly, all within the range of $5 \mathrm{~cm}$; under the binary system, deviations of the points were mainly in $2-5 \mathrm{~cm}$, of which 5 points showed the deviation above $5 \mathrm{~cm}$. In general, the triple-satellite system showed higher and more stable measurement accuracy.

In the end, 24 control points went through threedimensional unrestrained and restrained adjustment in CGCS2000 coordinates to obtain statistics of relative mean error of the weakest side and mean square error of the weakest point, as shown in Table 2:

Table 2 Statistical table of the relative median error of the weakest side and the median error of the weakest point

\begin{tabular}{|c|c|c|c|c|c|}
\hline \multicolumn{2}{|c|}{ project } & \multicolumn{2}{|c|}{ GPS+GLONASS+BDS } & \multicolumn{2}{|c|}{ GPS+GLONASS } \\
\hline \multirow{3}{*}{$\begin{array}{c}\text { Three - } \\
\text { dimensional } \\
\text { unconstrained } \\
\text { adjustment }\end{array}$} & \multirow[t]{2}{*}{$\begin{array}{c}\text { The weakest side relative mean } \\
\text { error }\end{array}$} & \multicolumn{2}{|c|}{$1 / 2133600$} & \multicolumn{2}{|c|}{$1 / 1948300$} \\
\hline & & $\mathrm{X} 、 \mathrm{Y}$ & $\mathrm{H}$ & $X, Y$ & $\mathrm{H}$ \\
\hline & $\begin{array}{l}\text { Median error of weakest point } \\
\qquad / \mathrm{cm}\end{array}$ & 0.523 & 1.221 & 0.625 & 2.014 \\
\hline \multirow{3}{*}{$\begin{array}{c}\text { Three } \\
\text { dimensional } \\
\text { constraint } \\
\text { adjustment }\end{array}$} & $\begin{array}{c}\text { The weakest side relative mean } \\
\text { error }\end{array}$ & \multicolumn{2}{|c|}{$1 / 2296800$} & \multicolumn{2}{|c|}{$1 / 2034900$} \\
\hline & & $\mathrm{X}, \mathrm{Y}$ & $\mathrm{H}$ & $X, Y$ & $\mathrm{H}$ \\
\hline & $\begin{array}{c}\text { Median error of weakest point } \\
\qquad / \mathrm{cm}\end{array}$ & 0.872 & 1.785 & 0.932 & 2.134 \\
\hline
\end{tabular}

According to Table 2, relative mean error of the weakest side of GPS+GLONASS+BDS and GPS+GLONASS modes met $1 \times 10-6$ as provided by $\mathrm{C}$ level control points' relative accuracy requirements. $\mathrm{X}$ and $\mathrm{Y}$ - relative mean error of the weakest point under GPS+GLONASS+BDS mode was $\leqslant 10 \mathrm{~mm}$, and $\mathrm{H} \leqslant$ $18 \mathrm{~mm}$. $\mathrm{X}$ - and Y- plane relative mean error under GPS+GLONASS mode was $\leqslant 10 \mathrm{~mm}$, and $20 \leqslant \mathrm{H} \leqslant$ $22 \mathrm{~mm}$. Therefore, GPS+GLONASS+BDS and GPS+GLONASS modes showed little difference in plane coordinates; elevation of GPS+GLONASS mode was nearly $2 \mathrm{~mm}$ higher than the specification; in $\mathrm{X}, \mathrm{Y}$ and $\mathrm{H}$, the precision of GPS+GLONASS+BDS was higher than that of GPS+GLONASS.

\section{Conclusion}

In this paper, observation data from Guangxi BeiDou GBAS base stations were resolved; by comparing the residuals of GPS tri-dimensional baseline vectors, deviation of three-dimensional coordinates, relative mean error of the weakest side and mean square error of the weakest point of C-level control points under the GPS+GLONASS+BDS mode and the GPS+GLONASS mode, the effect of data collected under different satellite combinations on measurement accuracy under different terrain conditions was analyzed. According to test results, the triple-satellite system including BDS is of higher and more stable measurement accuracy, and has more obvious advantages in complex mountainous areas.

\section{Reference}

1. GB/T18314-200 Specifications for global positioning system(GPS)surveys.

2. YANG Yi-fei, CHEN Xiang-ping, Research and Accuracy Evaluation of GNSS Automatic Solution Software. Geospatial Information 2019,17(04),25-28.

3. Yang Kunlun. Application Effect of BDS, GPS and GLONASS Single Satellite System in Engineering Control Network.Geomatics Technology and Equipment 2018,20(04),11-14.

4. YANG Kunlun, Comparison of BDS and GPS measurement data under adverse observation conditions.Bulletin of Surveying and Mapping 2019(S1):22-25.

5. CAO Baisong, Using the GAMIT to Do a CORSBased GNSS Static Data Processing.BeijingSurveying and Mapping 2019,33(7):802-806.

6. CHEN Xiangping. Performance Test and Accuracy Analysis of Guangxi BeiDou Ground-based Augmentation System.Geospatial Information 2019,17(07)65-67.

7. SUN Guopeng,LI Jiansheng ,HAO Xiangyang ,ZHANG Xiaodong, Comparing and Analyzing for Chinese GNSS Data Processing Software.GNSS World of China 2017,42(01),103-10. 